\section{Random Drift and Design Creativity: Evolution of Drawings in the Laboratory}

\author{
Minoru Matsui", Kenta Ono, Makoto Watanabe \\ Chiba University, 1-33 Yayoi-cho Inage-ku Chiba-shi Chiba 263-8522, \\ Japan \\ "Author for correspondence (xerroxcopy@gmail.com)
}

Previous cultural analyses argue that a randomcopying model that is analogous to genetic drift in population genetics explains a variety of real-world datasets. Few empirical investigations have been done on how cultural traits are actually generated and selected. We present experimental data that matches random-copying simulation very well. In our experiment, designers copied what they considered well designed, and eliminated the poor ones, and designed several novel drawings by different design strategies in a cultural transmission network. What were conventionally thought useful for design to prosper, such as practice, exposure to other design and experience in design, do not quite contribute to its prosperity. We suggest that some design's creation processes as well as its market may be value-neutral.

\section{Keywords}

cultural transmission, power-law distribution, cultural drift, cultural evolution, design creativity

\section{Introduction}

Previous studies have shown that simple random copying model represents various real-world cultural datasets, such as pop chart (Bentley, Lipo, Herzog, \& Hahn, 2007; Salganik, Dodds, \& Watts, 2006), patent and paper citations (Bentley, Hahn, \& Shennan, 2004; Simkin \& Roychowdhury, 2003), pottery types and decorations (Bentley et al., 2004; Shennan \& Wilkinson, 2001), and first names (Hahn \& Bentley, 2003). These studies suggest that no selection is needed to explain diverse variation of successful and non-successful cultural traits.

In the neutral theory of molecular evolution, gene frequencies changes only by chance and simply due to sampling error. We show that neutral model also represents a simple experimental design process, in which participants eliminate the former time-step line drawings and introduce the same amount of novel drawings in a cultural transmission network, that is similar to transmission chain practiced by Tamariz \& Kirby (2014).

By comparing cumulative frequency of drawing to that generated by random drift simulation very close to the condition of the experiment, we show that these two match very well. For mutation rate $\mu \ll 1$, previous studies show that real-world cultural variants and simulation datasets obey power-law distribution,

$$
p(x) \propto x^{-\alpha} .
$$

However, it is known that the pattern breaks down for large $\mu$ (Bentley et al., 2004). To make matters worse, finite size effect bends off the extreme lower tail. Hence, along with basic power-law distribution, we fitted powerlaw with exponential cut-off,

$$
p(x) \propto x^{-\alpha} e^{-\lambda x},
$$

which is characterized by power-law behaviour for $x$ $\ll \lambda^{-1}$ and exponential distribution behaviour for $x \gg \lambda^{-1}$.

\section{Methods}

\section{Participants}

Forty-two volunteers (age $M=23.6, S D=6.27$, range = 18-57 years, twenty-six of them were design students) participated, each representing one time-step $(t)$. Participants with and without design experience were interlaced in terms of time-step, to better represent actual design processes, in which designs are produced and evaluated by both professional and non-professional people, e.g., Wikipedia. Each participant designed six novel drawings, resulting in $n=252$ unique drawings in total.

\section{Procedure}

Design criteria are a list of purposes that design is expected to meet. Because our main interest was not in the effect of specific design criteria, they could have been anything. In our experiment, we chose the following four, to empower any participants including those who had no experience in design at all: to design drawings that they like; easy to copy; easy to remember; and stand out. Participants had to treat these criteria equally. Letters were prohibited to draw because it is known to be tenacious in transmission chain (Tamariz \& Kirby, 2014). Filling was also prohibited because the filled drawings were expected to stand out from the other drawings, while hatching was allowed. These criteria and restrictions were informed to the participants before drawing. The initial drawings were drawn by the authors.

Drawing process was done in the following order. On a draft sheet, a participant was asked to design total of six unique drawings in accordance with six different drawing strategies. When all were drawn on the draft sheet, the participant was asked to trace these six drawings to a sheet for a clean copy. Along with these, participants copied ten drawings from previous time-step, on an A4 white paper 
with sixteen $40 \mathrm{~mm}$ square drawing fields printed on, with $1.6 \mathrm{~mm}$-thick black ballpoint pen (see Figure 1 for example).

The clean copy was subsequently handed to the next participant. This process was repeated for the total timesteps of forty-two times.

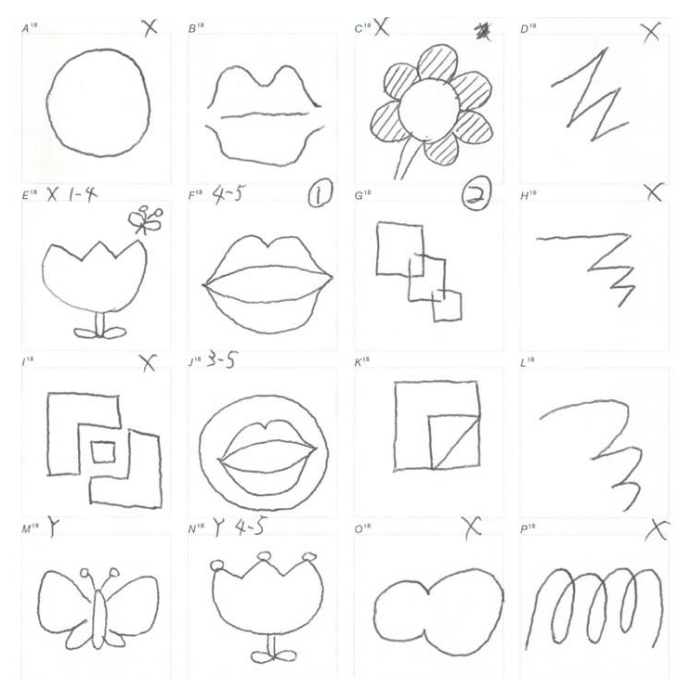

Figure 1. An example of a sheet, $t=18$. X-marks on the upper-right corners indicate that the drawings were eliminated from the population by the subsequent participant. 1 (on F18) and 2 (on G18) indicate the drawings were chosen as the best, and the drawing was chosen as the second best, respectively.

\section{Drawing strategies}

The six drawing strategies consist of two copying strategies ("Impr.P" and "Hybr.P") and four innovation strategies ("Pre," "Pre.P," "Post" and "Post.P"). For drawings with the suffix ".P," the drawer drew at least one practice drawings until $\mathrm{s} /$ he was satisfied with the result, and chose the best one out of them. The drawer was allowed to pick whichever trial.

After the instruction, the research participant drew the first drawing, Pre, without any practices. Then the drawer was prompted to draw Pre.P, with practice as explained above. After that, the drawer received the former timestep's clean copy sheet and chose the best two drawings, as well as the worst six. Based on the best drawing, the drawer was asked to draw the draft of third drawing, Impr. $\mathrm{P}$, improving the design even more. Then the drawer was asked to draw Hybr.P, a combination of the best and the second best drawing, hybridizing them, cherry-picking the good qualities that made the drawers choose them as the best two. This procedure was adopted to mimic the procedure of the random copying model setting of the simulation, in which a certain generation's variants have slight chance of copying the same variant from the previous time-step. Post drawing was drawn only once, just like Pre. The last drawing, Post.P, was with practice just like Pre.P. After drawing them, the drawer proceeded to trace the predecessor's drawings except the worst six. These vacant six fields were replaced by the novel six drawings.

\section{Simulation}

An R script equivalent to the infinite allele model for a single-locus, multiple neutral allele system (Kimura \&

Matsui et al. LEBS Vol. 8 No.2 (2017) 24-27
Crow, 1964) was used to simulate random copying model with similar parameters as the experiment. In the agentbased model simulation, unique cultural traits represented by arbitrarily defined numerical variants are assigned to a population of $N$ individual agents. Each agent then iteratively copies from the previous time-step randomly, with probability $(1-\mu)$, or innovates a novel trait, with probability $\mu$ (see Hahn \& Bentley, 2003; Mesoudi \& Lycett, 2009 for details). To match the conditions to our experiment in the lab, we ran simulations for $T=42$ timesteps with a population of $N=16$ agents. Simulation was run for 100 times each.

\section{Counting the frequency of drawing}

While there is a clear-cut difference between copy and mutation in random drift simulation, real world design process as well as our transmission network has blurry distinction. It is not straightforward to determine which mutations are actually novel and which are not. To overcome this, we defined frequency of design that corresponds to arbitrarily-assigned degree of influence on descents, or contribution ratio, $V(0 \leq V \leq 1)$.

When $V=0$, even a slightest modification of design is considered innovative. No parent-offspring relationships is taken into account. The frequency of design is determined simply by its cumulative number of copies throughout the experiment. Let us call this base frequency $f$. For $0<V<$ 1 , the parents split their descendants' frequency equally. In our case, an Impr.P drawing (which has one parent) acquires only $(1-V)$ of its cumulative frequency $f$. The rest, $V f$, is given to its parent. Similarly, Hybr.P drawing (two parents) gives $V f / 2$ to each of its two parents. When $V$ $=1$, This way we get frequency of a design $X$ as

$$
F_{V}(X)= \begin{cases}f(X) & \left(p_{X}=0\right) \\ (1-V) f(X) & \left(p_{X}=1,2, \ldots\right),\end{cases}
$$

where $p_{X}$ is the number of parents of $X$, and $f(X)$ is the frequency

$$
f(X)=f_{0}(X)+V \sum_{k=1}^{l} \frac{f\left(x_{k}\right)}{p_{x_{k}}},
$$

where $f_{0}(X)$ is the number of time-steps $X$ has survived, $x_{1}$, $x_{2}, \ldots, x_{\mathrm{i}}$ are the direct descendants of $X$ (if available). Note that $p_{x_{k}} \neq 0$ because $x_{\mathrm{k}}$ has at least one parent, $X$. This way the sum of $F(X)$ is a constant for any $V$,

$$
\sum_{i=1}^{n} F_{V}\left(X_{i}\right)=N \times T,
$$

where $n$ is the total number of designs generated through total of $T$ time-steps in $N$ individuals.

In our analysis, we considered three scenarios: $V=0$, 0.5 , and 1 . Corresponding mutation rates for simulation is $\mu=6 / 16,5 / 16$, and $4 / 16$. As V varies, frequency also varies greatly. As can be seen in Figure 2, individual $\mathrm{J}$ in timestep $t=1$, J1, had survived through three time-steps, thus $F_{0}(\mathrm{~J} 1)=3$, and similarly, $F_{0}(\mathrm{~L} 2)=13$, while $F_{1}(\mathrm{~J} 1)=20$ and $F_{1}(\mathrm{~L} 2)=0$.

\section{Analysis}

Discrete power-law distribution fits and tests were 


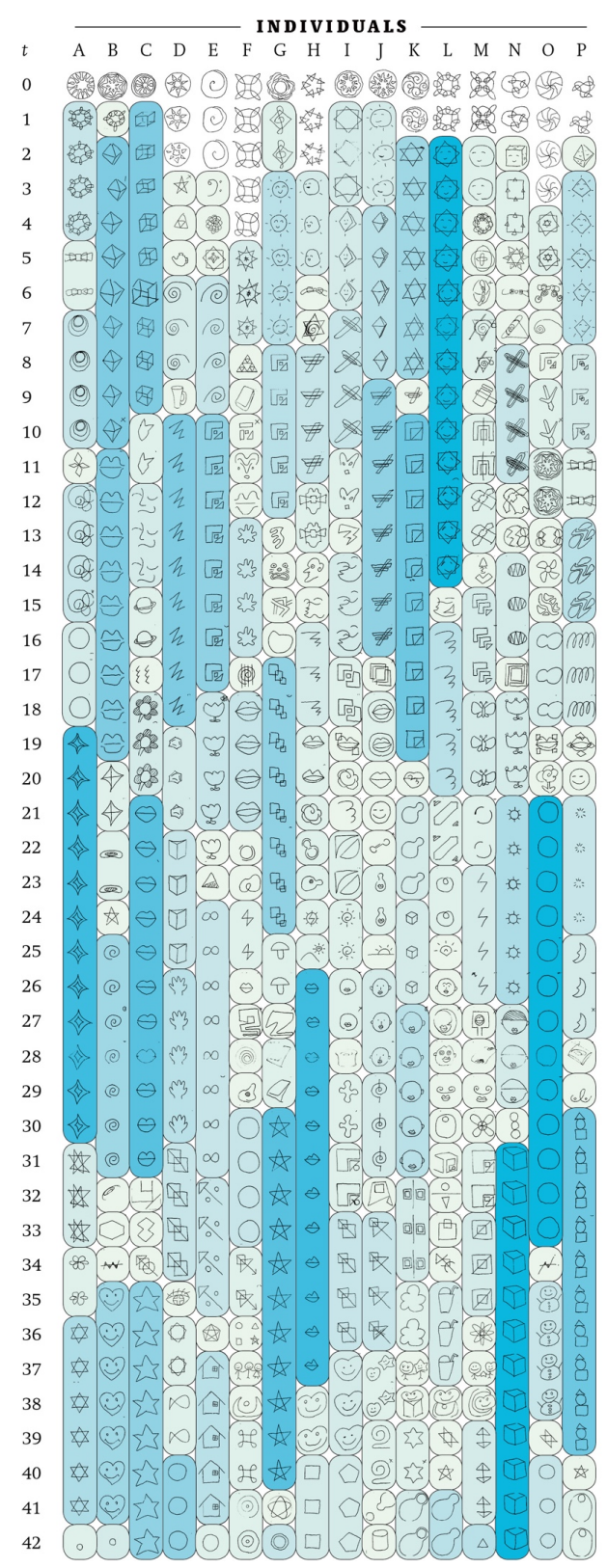

Figure 2. Experiment result. Each column represents an individual (A to P). Each row represents a successive timestep and the drawings designed by a participant. The same design is circled together. Colour thickness corresponds to the number of time-steps the design had survived.

performed in the $\mathrm{R}$ package poweRlaw (Gillespie, 2015) to estimate maximum likelihood power-law exponent $\alpha$ and to test its Goodness-of-fit with 1000 bootstrap runs. R scripts (Clauset, Shalizi, \& Newman, 2009) were used to fit power-law with cut-off.

We also fit a probabilistic model to the observed distributions. We assumed that frequency is affected by following factors: educational background, time-step, repetitive practice (prototyping), exposure to others' drawings, improving or combining the existing designs in the population. All rates were estimated using Bayesian Markov-chain Monte Carlo methods with R package Rstan (Stan Development Team, 2016).

\section{Results and Discussion}

As shown in Figure 3-[A] and [B], experimental result showed good fit to simulation outcome. On the other hand, Goodness-of-fit tests did not convince power-law was a plausible model either for simulation or experiment results. The $p$-values were below 0.1 for all $V \mathrm{~s}$ and most simulation runs $(97,96$, and 88 runs for $V=0,0.5$, and 1, respectively. In Goodness-of-fit test, the high $p$-value suggests power-law is a plausible fit). However, likelihoodratio tests showed that power-law with cut-off was good fit for experiment results for any $V_{\mathrm{S}}(p<0.01$, this time the smaller $p$-value indicates power-law with cut-off is better fit than basic power-law), as well as all random drift simulations ( $p<0.05$ for all runs). As can be seen in Figure 3-[B], the match between random drift model and experiment result became better as $V$ got close to 1 . In overall, the statistical support for the power-law hypothesis is poor both for experimental and simulation results, while power-law with cut-off is clearly favoured over the basic power-law, and the parameters of them match very well.

Contrary to our conventional understandings, Poisson regression revealed almost no effects of the following factors (Figure 3-[C]): educational background, repetitive practice, and combining the previous works. Time-step and exposure to others' drawings had negative effect. The only clear positive effect was found on improving the previous works, estimated to multiply frequency by 1.70 (95\% CI: 1.26-2.32) for $V=0$, and 1.014 (95\% CI: $0.73-1.42)$ for $V=$ 0.5 (note that if improving had no effect on frequency, the rate would have been around 0.5 because frequencies Impr. $\mathrm{P}$ acquired is multiplied by 0.5 for $V=0.5$, as we defined so).

The effect of improving, however, was not even close to the random effects of individual designs. The largest random effects of an individual design for each of the $V$-values were estimated to multiply frequency by 3.88 (95\% CI: 1.97-7.46) times, 4.46 (95\% CI: 2.56-7.24) times, and 14.2 (95\% CI: 9.33-21.83) times for $V=0,0.5$, and 1 , respectively.

It is shown that markets for artificially designed cultural traits are often random and therefore unpredictable (e.g., Salganik et al., 2006). Our result extends this understanding that some design creation processes are, in fact, value-neutral: the main cause of a few very successful design in real-world may also be explained by simple model of random drift. Heavily depending on designers' and consumers' subjective preferences, many of our design creation and selection might not affect the value of design ideas as much as we have typically thought.

Our research was entirely executed in the laboratory. It is highly possible that some of the actual design processes are not value-neutral. Nonetheless, we think that this method is one of the few feasible approaches to tackle yetto-be-discovered mechanisms of design creation and its selection.

\section{References}

Bentley, R. A., Hahn, M. W., \& Shennan, S. J. (2004). Random drift and culture change. Proceedings of the Royal Society of London B: Biological Sciences, 271, 1443-1450. (doi: 10.1098/rspb.2004.2746)

Bentley, R. A., Lipo, C. P., Herzog, H. A., \& Hahn, M. W. 

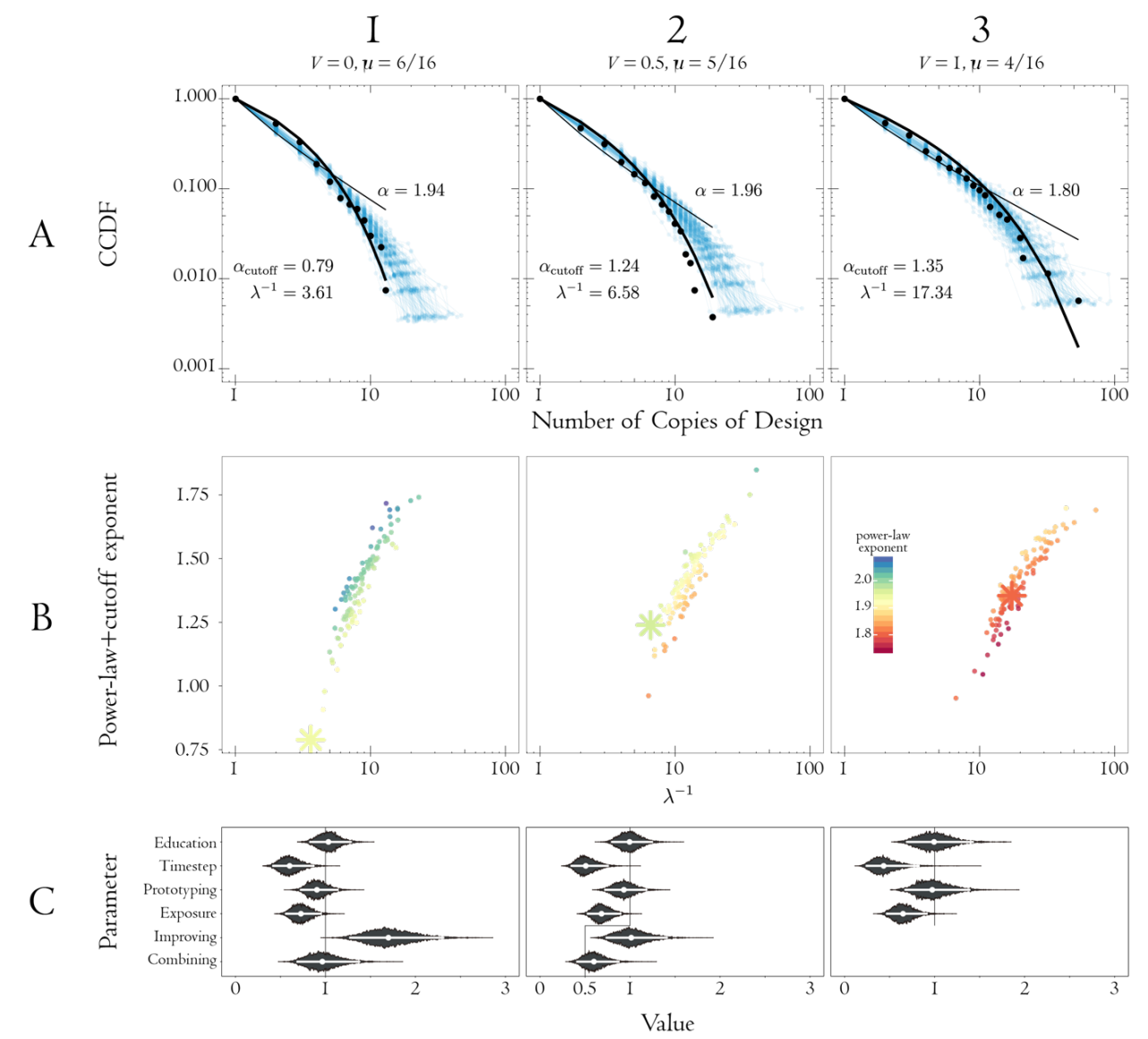

Figure 3. Columns from left to right: $V=0,0.5,1$. Row [A]: Power-law distributions of the experiment (black) compared to outcome of simulation (light blue). Complementary cumulative distribution function $P(x)$ value on the $y$-axis, frequency of a drawing on the $x$-axis. Since the discrete power-law fit only accepts integers, frequencies for $V>$ 0 were rounded. Maximum likelihood power law (line) and that with exponential cut-off (thick curve) along with their parameters are also plotted. Row [B]: Maximum likelihood parameters of power-law with cut-off. Exponent $\alpha_{\text {cutoff }}$ on $y$-axis, rate $\lambda^{-1}$ on $x$-axis. Simulation runs with dots, asterisk for experiment result. Colour corresponds to the exponent of basic power-law without cut-off. Row [C]: Estimated posterior distribution of coefficients for various parameters, with a point on median of the estimation. The line indicates $95 \%$ Bayesian confidence interval. Parameters from top to bottom: the effect of educational background ( 1 if the drawer is a design student), time-step (divided by total time-steps), prototyping (scaled to the effect of 10 prototypings), improving (1 for Impr.P), combining (1 for Hybr.P), and exposure to previous time-step's drawings (1 for Impr.P, Hybr.P, Post, and Post.P).

(2007). Regular rates of popular culture change reflect random copying. Evolution and Human Behavior, 28, 151-158. (doi: 10.1016/j.evolhumbehav.2006.10.002)

Clauset, A., Shalizi, C. R., \& Newman, M. E. J. (2009). Power-law distributions in empirical data. SIAM Review, 51, 661-703. (doi: 10.1137/070710111)

Gillespie, C. S. (2015). Fitting heavy tailed distributions: the poweRlaw package. Journal of Statistical Software, 64. (doi: 10.18637/jss.v064.i02)

Hahn, M. W., \& Bentley, R. A. (2003). Drift as a mechanism for cultural change: an example from baby names. Proceedings of the Royal Society of London B: Biological Sciences, 270, S120-S123. (doi: 10.1098/ rsbl.2003.0045)

Kimura, M., \& Crow, J. F. (1964). The number of alleles that can be maintained in a finite population. Genetics, 49, 725-738.

Mesoudi, A., \& Lycett, S. J. (2009). Random copying, frequency-dependent copying and culture change. Evolution and Human Behavior, 30, 41-48. (doi: 10.1016/j.evolhumbehav.2008.07.005)

Salganik, M. J., Dodds, P. S., \& Watts, D. J. (2006).

Matsui et al. LEBS Vol. 8 No.2 (2017) 24-27
Experimental study of inequality and unpredictability in an artificial cultural market. Science, 311, 854-856. (doi: 10.1126/science.1121066)

Shennan, S. J., \& Wilkinson, J. R. (2001). Ceramic style change and neutral evolution: a case study from Neolithic Europe. American Antiquity, 66, 577-593. (doi: 10.2307/2694174)

Simkin, M. V., \& Roychowdhury, V. P. (2003). Copied citations create renowned papers? arXiv.org e-Print archive: cond-mat/0305150 (Annals of Improbable Research, 11, 24-27, 2005, Retrieved from http:// www.improbable.com/airchives/paperair/volume11/ v11i1/v1li1.html (last accessed on May 8 2017))

Stan Development Team. (2016). RStan: the R interface to Stan. R package version. Retrieved from http://mcstan.org (last accessed on May 8 2017)

Tamariz, M., \& Kirby, S. (2014). Culture: copying, compression, and conventionality. Cognitive Science, 39, 171-183. (doi: 10.1111/cogs.12144) 